\title{
Power Consumption Evaluation of Efficient Digital Signature Schemes for Low Power Devices
}

\author{
Stefaan Seys and Bart Preneel \\ K.U.Leuven, Department Electrical Engineering-ESAT, SCD/COSIC \\ Kasteelpark Arenberg 10, B-3001 Leuven, Belgium \\ email: \{stefaan.seys, bart.preneel \} desat.kuleuven.ac.be
}

\begin{abstract}
In this paper we evaluate the power consumption of different digital signature schemes. We compare the cost of the Elliptic Curve Digital Signature Algorithm with signature schemes solely based on symmetric techniques such as the DiffieLamport one-time signature scheme. These evaluations take into account all aspects of using digital signatures in wireless environments: energy consumption of key generation, signing and verification, and the communication cost of sending and receiving the necessary data (including the public keys and the necessary data to authenticate them).
\end{abstract}

\section{INTRODUCTION}

The world of communicating electronics can be separated into wired and wireless devices. While the former typically do not suffer from power restrictions, wireless devices have limited energy resources (for example the battery of a cellular phone) and are restricted in power usage. The recent advance in micro electro-mechanical systems and wireless communication technology makes it a pragmatic vision to deploy large-scale, low power, inexpensive distributed sensor networks (DSNs) [1], [2]. During the past few years, a substantial effort has been directed to turn this vision into reality. Research prototype sensor nodes [3], [4] are being designed and manufactured, energy efficient Medium Access Control protocols [5], topology control protocols and routing schemes are implemented and evaluated [6]; various enabling techniques such as time synchronization, localization and tracking are being studied. Security related research has been directed towards "efficient" or "low-power" protocols for authentication [7], [8], key management and other related areas. But only limited research has been done on the power consumption of the underlying cryptographic primitives, such as block ciphers, one-way functions, digital signatures, etc. on which these schemes are based.

In this paper we focus on the power consumption of digital signature algorithms based solely on symmetric primitives. These digital signature are presented as efficient and fast and are assumed to be less power consuming than schemes based on expensive operations such as modular exponentiation. To our best knowledge, these claims are not supported by any proof or actual simulation that includes all aspects of using these schemes in wireless environments: energy consumption of key generation, signing and verification, and the communication cost of the signatures and the required

This work was supported by the Concerted Research Action (GOA) Ambiorics 2005/11 of the Flemish Government.

Stefaan Seys is financed by a Ph.D. grant of the the Institute for the Promotion of Innovation through Science and Technology in Flanders (IWTVlaanderen). authentication data. We present a detailed analysis of two one-time signature schemes (the Lamport-Diffie scheme [9] and the HORS scheme [10]) together with two public key authentication mechanisms that make it possible to turn these one-time schemes into "real" digital signature algorithms. We compare the power consumption of these schemes with the power consumption of the Elliptic Curve Digital Signature Algorithm (ECDSA) [11].

\section{A. Hardware}

After providing generic expressions for the costs of the different signature schemes, we compare them with ECDSA using the power measurements of A. Hodjat and I. Verbauwhede on a Wireless Integrated Network Sensor (WINS) sensor [12]. WINS is a StrongARM based wireless sensor which was developed at Rockwell Scientific [13]. It has an Intel StrongARM SA1100 embedded controller, which is a general-purpose, 32-bit RISC microprocessor based on the ARM architecture that is rated the most efficient processor (in MIPS/Watt). The processor offers a $16 \mathrm{~KB}$ instruction cache, an 8KB data cache, serial I/O and JTAG interface all combined in a single chip. Program and data storage are provided by $1 \mathrm{MB}$ SRAM and $4 \mathrm{MB}$ of bootable flash memory. Embedded firmware has been developed to support multiple access networking with minimal ARM processor support. The radio is capable of operating at multiple transmit power levels between 1 and $100 \mathrm{~mW}$ enabling the use of power-optimized communication algorithms. It can be equipped with additional sensing modules such as an acoustic sensor, a magnetometer, an accelerometer or a seismic sensor. The whole is packaged in a $90 \times 90 \times 75 \mathrm{~mm}$ container.

A. Hodjat and I. Verbauwhede measured the energy consumption of the AES secret-key encryption/decryption and the elliptic curve point-multiplication (ECPM) algorithms. They also investigated the power consumption of the radio transmission. The results relevant to this paper are summarized in Table I. Notice that the cost of a AES encryption with a 128-bit key is three orders of magnitude smaller than one ECPM.

Commercial button cell battery energy densities range from $1000-2500 \mathrm{~J} / \mathrm{cm}^{3}$. Using the numbers in Table I we see that with this setup it is possible to perform 3 to 8 million AES 128-bit block encryptions per $\mathrm{cm}^{3}$ of battery volume and only 1000-2500 ECPMs in a 192-bit field.

\section{B. Conventions used in this Presentation}

All the one-time signature and public-key authentication schemes evaluated in this paper are based on a general one- 
TABLE I

POWER CONSUMPTIONS OF THE AES ALGORITHM AND ELLIPTIC CURVE POINT-MULTIPLICATION ON A WINS NODE. THE ENERGY COST OF THE AES ALGORITHM INCLUDES KEY SCHEDULING.

\begin{tabular}{|l|c|c|c|}
\hline \multirow{2}{*}{$\begin{array}{l}\text { AES block cipher } \\
\text { (key = 128 bit) }\end{array}$} & \multicolumn{3}{|c|}{ block size } \\
\cline { 2 - 4 } Encryption (mJoules) & $\mathbf{1 2 8}$ bit & $\mathbf{1 9 2}$ bit & $\mathbf{2 5 6}$ bit \\
\hline Decryption (mJoules) & 0.31 & 0.38 & 0.42 \\
\hline \hline Elliptic Curve & 0.36 & 0.62 & 1.01 \\
\hline point-multiplication & \multicolumn{3}{|c|}{ Galois field size $\left(2^{n}\right)$} \\
\cline { 2 - 4 } Power consumption (mJoules) & $\mathbf{1 2 8}$ bit & $\mathbf{1 9 2}$ bit & $\mathbf{2 5 6}$ bit \\
\hline \hline Radio communications (@ 100 kbit/s) & 1070 & 2340 \\
\hline $\begin{array}{l}\text { Transmission mode } \\
\text { Receive mode }\end{array}$ & $1080 \mathrm{mWatt}$ or $10.80 \mu \mathrm{J} / \mathrm{bit}$ \\
\hline
\end{tabular}

way function (OWF) $f($ ). In order to be able to provide algebraic expressions for the cost (= power requirement) of these schemes we assume that:

- the input size of $f()$ is a multiple of $n$ bit; we will refer to these $n$ bit as one "block";

- the output size of $f()$ is 1 block;

- the cost of $f()$ for an input size of $t$ block is $t$ BF (for Block Function), i.e., linear with respect to the input size. In order to compare the cost of the one-time signature schemes with ECDSA, we assume that the OWF $f()$ is based on AES encryption with a 192-bit block size.

For all signature schemes discussed in this paper, we assume that the message that is signed has already been hashed by a cryptographic hash function, resulting in a $s$-bit output. Examples of such a cryptographic hash function are SHA-1 and RIPEMD-160 [14].

\section{Outline}

First, we evaluate the cost of ECDSA, the Diffie-Lamport one-time signature scheme and the HORS one-time signature scheme. Next we compute the energy consumption of these schemes when they are turned into general purpose signature schemes using two different authentication mechanisms. Finally we compare the efficiency of the different aspects of these schemes (communications, signature generation and verification) using the numbers provided in Table I.

\section{Elliptic Curve Digital Signature Algorithm}

The Elliptic Curve Digital Signature Algorithm (ECDSA) [11] is the elliptic curve analogue of the Digital Signature Algorithm (DSA) [14]. It was accepted in 1999 as an ANSI standard, and was accepted in 2000 as IEEE and NIST standards. The ECDSA signature generation requires 1 ECPM, 1 integerinversion, 2 integer-multiplications and 1 SHA-1 invocation on the message. Verification requires an additional ECPM. From [15, chapter $V]$ we learn that the point-multiplication $k P$ requires around 10 integer-multiplications for every bit in $k$. This means that the cost for a ECDSA signature generation (verification) is approximately one (two) ECPMs plus the SHA-1 hash computation.

\section{LAMPORT-DifFIE ONE-TIme Signatures}

The Lamport-Diffie one-time signature [9] is based only on the concept of one-way functions. In its basic form it can be used to sign a single bit of data. The public key $y$ is computed from the random private key $x$ as follows: $y=f(x)$. The verifiers receive an authenticated copy of $y$. In order to sign a single bit $b$, the signer either reveils $x$ (sign bit "1") or does not reveil $x$ (sign bit "0"). The only way that the verifier can ever obtain $x$ is because the signer has revealed it to him; hence the verifier can prove that the signer has signed bit " $b$ ". Obviously extra precautions need to be taken to prevent the verifier from denying having received the value $x$ (see below).

\section{A. Basic Scheme}

The signature scheme above can be used to sign a single bit of data. This scheme can be extended to sign an $s$-bit message $m$ :

1) Generate a random private key $s k=\left\{x_{0}, \ldots, x_{s}\right\}$,

2) compute the public key $p k=\left\{f\left(x_{1}\right), f\left(x_{2}\right), \ldots, f\left(x_{s}\right)\right\}$ and transfer an authenticated copy to the verifier,

3 ) if the $j$ th bit of $m$ was 1 then reveal $x_{j}$, else do not reveal $x_{j}$.

Note that the recipient can always deny that she ever received some $x_{i}$ and hence change a bit of the message from a " 1 " into a "0". This problem can easily be solved by padding the message $m$ with the value $s-h$ in binary form, with $h$ the Hamming weight of $m$. This requires adding $\left\lceil\log _{2}(s)\right\rceil$ bits of redundancy to the original $s$-bit message [16].

Generating the public key requires $s+\left\lceil\log _{2}(s)\right\rceil$ BF. On average the message and padded redundancy will contain an equal amount of zeros and ones. This means that the recipient only has to perform $\frac{1}{2}\left(s+\left\lceil\log _{2}(s)\right\rceil\right)$ BF to verify the signature. A signed message consists of the message $m$, $s+\left\lceil\log _{2}(s)\right\rceil$ random values $y_{j}=f\left(x_{j}\right)$, and $\frac{1}{2}\left(s+\left\lceil\log _{2}(s)\right\rceil\right)$ disclusures of $x_{j}$.

It is easy to realize that the Lamport-Diffie scheme becomes unmanageble as the number of messages that has to be signed grows. When signing $k$ messages using this scheme, we need to compute the public key

$$
\mathbf{Y}=\left\{\begin{array}{c}
Y_{1}=y_{1,1}, y_{1,2}, \ldots, y_{1, s} \\
Y_{2}=y_{2,1}, y_{2,2}, \ldots, y_{2, s} \\
\vdots \\
Y_{k}=y_{k, 1}, y_{k, 2}, \ldots, y_{k, s}
\end{array}\right.
$$

and transfer an authenticated copy of it to the intended recipients. For $s=168$ and $k=1000, \mathbf{Y}$ will be 2.6 MByte.

\section{B. The Winternitz Improvement}

Winternitz suggested an improvement that reduces the size of the signature at the cost of additional computations [16]. In the Winternitz scheme we compute the value $y$ by applying $f()$ repeatedly, for example $y=f^{15}(x) .{ }^{1}$ This allows us to sign 4 bits of information with the single value $y$. To sign the four bit message 1001 (9 in decimal), the signer makes $\sigma=f^{15-9}(x)$ public. Anyone can check that $f^{9}(\sigma)=f^{9}\left(f^{15-9}(x)\right)=y$, thus confirming that $f^{15-9}(x)$ was made public, but no one besides the signer could have generated this value. Again extra redundancy has to be added to the signature in order to prevent people from changing the signature on 1001 into a signature on for example 1000 (8 in decimal) by computing $f\left(f^{15-9}(x)\right)=$ $f^{15-8}(x)$.

\footnotetext{
${ }^{1}$ Note that $f^{2}()=f(f())$ is applying the OWF $f()$ twice iteratively.
} 
In order to sign a $s$-bit message $m$, we first split $m$ in $s / g$ parts of size $g$ bits. Let these parts be $m_{1}, \ldots, m_{s / g}$. The secret key is $s k=\left\{x_{0}, \ldots, x_{s / g}\right\}$. The public key is $p k=\left\{f^{\left(2^{g}-1\right) s / g}\left(x_{0}\right), f^{2^{g}-1}\left(x_{1}\right), \ldots f^{2^{g}-1}\left(x_{s / g}\right)\right\}$. The signature of a message $m$ is computed by considering the integer value of the blocks $\operatorname{Int}\left(m_{i}\right)=I_{i}$. The signature $\operatorname{Sig}(m)$ is composed of $m / g+1$ values $\left\{\sigma_{0}, \ldots, \sigma_{s / g}\right\}$ where, for $i \geq 1, \sigma_{i}=f^{2^{g}-1-I_{i}}\left(x_{i}\right)=f^{-I_{i}}\left(y_{i}\right)$, while $\sigma_{0}=$ $f^{\sum_{i} I_{i}}\left(x_{0}\right)$ for $1 \leq i \leq s / g$. The signature length is $l(s / g+1)$. To verify a signature, again one splits the message $m$ in $s / g$ blocks of size $g$ bits. One then verifies that $p k$ equals $\left\{F^{2^{g}-1-\sum_{i} I_{i}}\left(\sigma_{0}\right), F^{I_{1}}\left(\sigma_{1}\right), \ldots, F^{I_{s / g}}\left(\sigma_{s / g}\right)\right\}$ for $1 \leq i \leq$ $s / g$. On average, both computing and verifying a signature require $(s / g)\left(2^{g}-1\right)$ evaluations of $f()$.

We see that the computational cost grows exponentially with $g$, while the communication cost only drops linear with $g$, this indicates that any performance gain, if any, will only be possible for small values of $g$. Table II shows the total cost of a signature for different values of $g$ (based on Table I). This cost includes public key generation, signature generation and one signature verification. The minimum cost occurs for $g=2$, i.e., signing 2 bits with a single value $y$, and the gain compared to the basic Lamport-Diffie scheme is about $20 \%$.

TABLE II

COST OF THE WINTERNITZ SIGNATURE SCHEME FOR DIFFERENT $g$ BLOCK SIZES. THE UNIT IN BOTH CASES IS mJoules.

\begin{tabular}{c|c|c|c|c|c|c} 
g & 1 & 2 & 3 & 4 & 5 & 6 \\
\hline $\mathbf{1 2 8}$ bit & 575 & 487 & 590 & 840 & 1307 & 2148 \\
$\mathbf{1 9 2}$ bit & 809 & 650 & 759 & 1056 & 1624 & 2651
\end{tabular}

\section{The HORS ONE-TIME SignATURE SCHEME}

\section{A. Description}

In this section we will focus on the one-time signature scheme of Reyzin and Reyzin [10] because to the best of our knowledge this scheme is the fastest and cheapest. More specifically, we will use their construction based on "subsetresilient" functions.

1) Short overview of the protocol: First the signer generates a private key $s k$ that consists of $t$ random values. The public key $p k$ is computed by applying a OWF $f()$ to each of the values of the private key. The public key $p k$ is distributed to the intended receivers in an authenticated way. A message is signed as follows: (1) use a cryptographic hash function $H$ to convert the message to a fixed length output, (2) use this output to uniquely select a subset $\sigma$ of maximum $k$ different values out of the set $s k$. This $\sigma$ is the signature for message $m$. The verifiers recompute the hash value of the message $m$, go through the same selection process to obtain a subset $\omega$ of the set $p k$ (instead of $s k$ ), and verify that $f\left(\sigma_{i}\right)=\omega_{i}$ for $1 \leq i \leq k$. Accept the signature if this is true for all $k$ values. It should be clear that this is a one-time signature scheme in the sense that with every signature $\sigma$ that is broadcast, part of the private key is leaked out. The security of this signature scheme is based on the one-wayness of $f()$ and on the assumption that, given a message $m$ and its signature $\sigma$, it is impossible to find another message $m^{\prime}$ that maps to the same $\sigma$. Note that this is a stronger assumption than $2^{\text {nd }}$-preimage resistance of the hash function $H$ (also referred to as weak collision resistance).
2) Detailed description: Let $f()$ be a one-way function and $H$ a cryptographic hash function with an $s$-bit output.

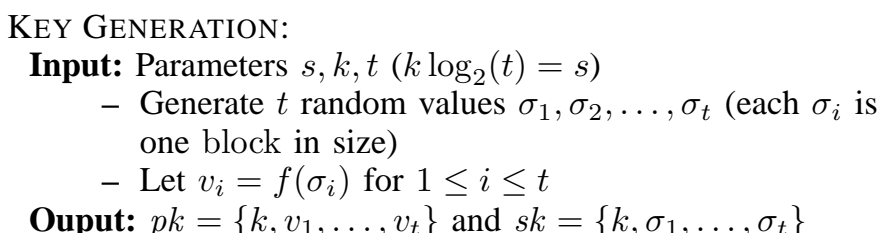

SIGNING:

Input: Message $m$ and secret key $s k$

- Let $h=H(m)$

- Split $h$ into $k$ substrings $h_{1}, h_{2}, \ldots, h_{k}$, of length $\log _{2}(t)$ bit each

- Interpret each $h_{j}$ as an integer $i_{j}$ for $1 \leq j \leq k$

Ouput: Signature $\sigma=\left\{\sigma_{i_{1}}, \sigma_{i_{2}}, \ldots, \sigma_{i_{k}}\right\}$

\section{VERIFYING:}

Input: Message $m$, signature $\sigma=\left\{\sigma_{1}^{\prime}, \sigma_{2}^{\prime}, \ldots, \sigma_{k}^{\prime}\right\}$ and the public key $p k$

- Let $h=H(m)$

- Split $h$ into $k$ substrings $h_{1}, h_{2}, \ldots, h_{k}$, of length $\log _{2}(t)$ bits each

- Interpret each $h_{j}$ as an integer $i_{j}$ for $1 \leq j \leq k$

Ouput: "accept" if for each $j, 1 \leq j \leq k, f\left(\sigma_{j}^{\prime}\right)=v_{i_{j}}$; "reject" otherwise.

\section{B. On the Security of HORS}

For this analysis we assume that the hash function $H$ behaves like a random oracle and that the adversary obtained signatures on $r$ random messages using the same private key. The probability that an adversary is able to forge a signature on a new message (without inverting the OWF $f()$ ) is at most $(r k / t)^{k}$. This is the probability that after $r k$ elements of $s k$ have been made public, $k$ elements are chosen (at random) that are a subset of them. The parameters $k, t$ and $s$ cannot be chosen independently, but have to satisfy $s=k \log _{2}(t)$. Using this, the probability can be rewritten as $2^{-\Sigma}$ with

$$
\Sigma=k\left(s / k-\log _{2}(k)-\log _{2}(r)\right) .
$$

As an example, for $s=160, k=16$ and $r=1$ (and $t=$ $1024)$, the security level is $2^{-96}$; for $r=4$ this probability rises to $2^{-64}$. In order to maximize efficiency, the number of signatures $r$ per public key should be as high as possible; while the security of the signature scheme drops with $r$. For now we will fix the security level of the signature scheme to $\bar{\Sigma}$. From this $\bar{\Sigma}$ we can derive the number of public keys $(\# p k)$ we need to sign $S$ messages:

$$
\# p k=S / r=S k 2^{(\bar{\Sigma}-s) / k} .
$$

Fig. 1 shows how \#pk changes with the parameter $k$ if we fix parameters $s=160$ and $S=2^{16}$, and $\Sigma=40$. We see that the number of public keys we need grows exponentially with $k$, but at the same time the size $t$ of each public key becomes smaller. As a practical example we will use the parameter set $(s, k, t, r)=(160,20,256,2)$ in the rest of this discussion, resulting in a security level of $\Sigma=53$. 


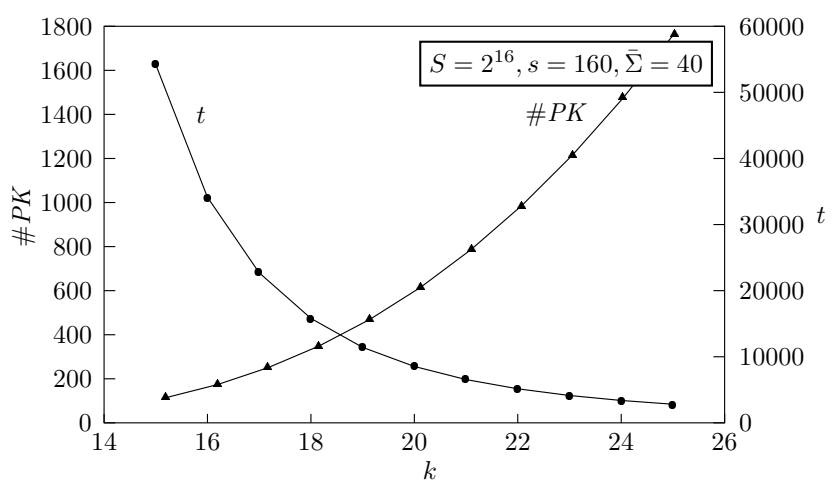

Fig. 1. Number of public keys and the size $t$ of each public key needed to sign $2^{16}=65536$ messages with the HORS signature scheme in function of the parameter $k$. The parameter $s$ is fixed to 160 and the security level $\Sigma$ was set to 40

\section{Cost Evaluation of HORS}

We assume that the system-wide parameter $k$ is fixed, and is not included in the public/secret key. The cost of HORS can be summarized as follows:

- Key generation requires $t$ evaluations of $f()$, resulting in a total computational cost of $t$ BF. The public key size is $t$ blocks.

- Signing requires no additional operations besides applying the hash function to the message. The signature size is $k$ blocks.

- Verifying costs a maximum of $k \mathrm{BF}$ if the signature is correct (if the signature is incorrect the verification process can be stopped earlier and the cost will be less).

\section{Merkle Hash Trees}

One of the disadvantages of the previously discussed onetime signature schemes is the size of the public key. All possible verifiers need an authenticated copy of this public key and store it in memory or receive a fresh authenticated copy together with every signature. Both alternatives are not very practical in most situations. This is where Merkle trees come in.

Merkle trees [16] can be used to efficiently authenticate $N$ values, with $N$ some power of two. These values are placed at the leaves of a binary tree. Fig. 2 is an example of a Merkle tree with $N=8$ leaves. Usually these values need to be kept secret; in that case the results of a OWF of these values are placed at the leaves of the tree. The values leaf ${ }_{i}=f$ (value) are called the leaves and the secret values are usually called the leaf pre-images. We will use the function PRECALC $(i)$ and $L E A F C A L C(i)$ to describe the process of creating the leaf pre-images and the leaves of the tree. The leaves are indexed from 0 to $N-1$. The parent's node value is calculated as a OWF $f()$ of the concatenation of the two child values. We use the following notation to describe the tree nodes:

$$
\begin{gathered}
\text { leaf }_{i}=P[i, i]=\operatorname{LEAFCALC}(i), \\
P[i, j]=f(\langle P[i,(i+j-1) / 2], P[(i+j+1) / 2, j]\rangle) .
\end{gathered}
$$

The height $H$ of the tree is defined as the number of levels in the tree, from the leaves to the root, i.e., $H=\log _{2}(N)$.

Since node $P[i, j]$ is a function of leaf ${ }_{i}$, leaf ${ }_{i+1}, \ldots$, leaf ${ }_{j}$, it can be used to authenticate these leaves. This implies that

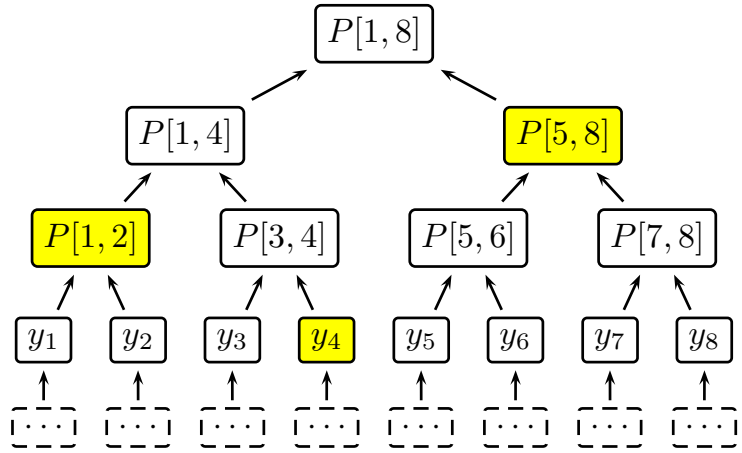

Fig. 2. Example Merkle hash tree with 8 leaves. The root node $P[1,8]$ can be used to authenticate the complete tree.

the whole tree is authenticated by the single root node of the tree. For example, root node $P[1,8]$ in Fig. 2 can be used to authenticate the leaves 1 through 8 . Assume that we wish to authenticate $l e a f_{3}$ to user $U$. User $U$ already obtained (out of band) an authenticated copy of the root node $P[1,8]$. The minimum information we have to send to $U$ is leaf $f_{3}$ itself, leaf $f_{4}, P[1,2]$ and $P[5,8]$. These values (colored gray in Fig. 2) allow $U$ to recreate the path from leaf ${ }_{3}$ to the root node. In general $\log _{2}(N)$ intermediate tree nodes, called the "authentication path", are needed to authenticate a leaf. Table III gives the authentication paths for a Merkle tree with 8 leaves. Note that most neighboring leaves share a large portion of their authentication paths.

\section{A. Cost Evaluation of Merkle Trees}

In order to evaluate the cost of Merkle trees we first describe the different processes involved.

- PRECALC generates the leaf pre-images and requires precost $\mathrm{BF}$.

- LEAFCALC generates the leaves and requires leafcost BF.

- Root generation is the process of computing the root node of the tree. This root node will serve as the public key of the signature scheme. We assume that root generation is done off-line on a powerful machine.

- Authentication path generation or Merkle tree traversal is the task that generates the authentication path for successive leaves.

Next to these steps we also consider the signing and verifying processes:

- Signing a message consists of two steps: (1) computing the signature (for example using the Diffie-Lamport scheme), and (2) generating the authentication path.

- Verifying a signature consists of (1) verifying the authenticity of the received leaf or leaves (i.e., the public key), and (2) verifying the signature.

1) Authentication path generation and verification cost: In [16], Merkle presents a straightforward technique that requires a maximum of $2 \log _{2}(N)-2$ evaluations of $f()$ per round ${ }^{2}$, and maximum space of $\frac{1}{2}\left(\log _{2}(N)\right)^{2}$ memory units (each unit the size of the output of $f())$.

\footnotetext{
${ }^{2}$ One evaluation of $f()$ costs $2 \mathrm{BF}$.
} 
TABLE III

AUthentication PATHS FOR A MerkLE TREe OF SIZE $n=8$ (DUPLICATE ENTRIES HAVE BEEN REMOVED).

\begin{tabular}{c|rrrr}
\hline LEAF & \multicolumn{3}{|c}{ AUTHENTICATION PATH } \\
\hline$Y_{1}$ & $P[1,8]$ & $P[5,8]$ & $P[3,4]$ & $P[2,2]$ \\
$Y_{2}$ & & & & $P[1,1]$ \\
$Y_{3}$ & & & $P[1,2]$ & $P[4,4]$ \\
$Y_{4}$ & & & & $P[3,3]$ \\
$Y_{5}$ & & $P[1,4]$ & $P[7,8]$ & $P[6,6]$ \\
$Y_{6}$ & & & & $P[5,5]$ \\
$Y_{7}$ & & & $P[5,6]$ & $P[8,8]$ \\
$Y_{8}$ & & & & $P[7,7]$ \\
\hline
\end{tabular}

In [17], a new algorithm (fractal Merkle tree traversal) is presented that allows a time-space trade-off. Briefly explained, the algorithm splits the original tree into subtrees of height $h \leq H$. These subtrees are constructed in such a way that the root of one tree is at the same time a leaf of a tree above it. Fig. 3 is a visualization of this. We call $L=H / h$ the number of subtree levels. Exactly one such subtree for each level is kept into memory and all these stacked subtrees together contain the authentication path for the leaf that is being authenticated at the moment. For each output of an authentication path a second new subtree for each level is being constructed. The construction is programmed in such a way that the new subtree will be finished right in time to be used to create the authentication path; at this moment the old subtree is discarded and the construction of a fresh subtree is initiated. Using the notation of Fig. 3 fractal tree traversal

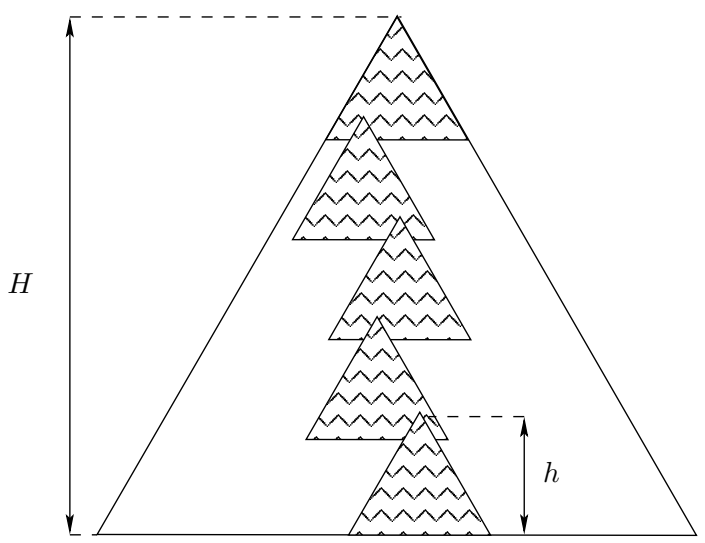

Fig. 3. Visualization of the fractal tree traversal algorithm described in [17].

requires a maximum of $2(L-1)$ evaluations of $f()$ per round, and maximum space of $2 L 2^{h+1}+H L / 2$ memory units (each unit the size of the output of $f())$.

Taking into account the cost of generating the leaves (leafcost $\mathrm{BF}$ ), the computational cost of fractal tree traversal becomes $(L-1)(2+$ leafcost $) \mathrm{BF}$ per round. The required space is minimized using $h=\log _{2}(H)=\log _{2}\left(\log _{2}(N)\right)$. The authentication path generation cost then becomes

$$
\frac{\log _{2}(N)}{\log _{2}\left(\log _{2}(N)\right)}(2+\text { leafcost }) \text { BF per round. }
$$

Upon receipt of a leaf pre-image and the corresponding authentication path, the recipients have to compute nodes of the tree until they arrive at the root. The total cost of this verification process is

$$
2 \log _{2}(N)+(\text { leafcost }- \text { precost }) \mathrm{BF} .
$$

\section{B. Cost Evaluation of Lamport-Diffie Signatures using Merkle} Trees

We propose the following scheme to use Merkle trees for authentication of the Diffie-Lamport public keys:

- Using the Winternitz improvement with parameter $g$, the private key consists of $s / g$ random values. These secret values $x_{l}$ are generated using a random seed value $K$ as follows: $x_{l}=A E S_{K}[l]$, with $l=0, \ldots, s / g-1$ for the first private key, $l=s / g, \ldots, 2 s / g-1$ for the second set, etc. The public key values $y_{l}$ are computed as described in Section III-B. Every public key is a leaf pre-images of the Merkle tree. Computing these leaf pre-images costs precost $=(s / g)\left(2^{g+1}-1\right) \mathrm{BF} .^{3}$

- The leaves themselves are the result of the OWF $f()$ applied to one of the public keys. This means that computing a leaf from a leaf pre-image costs $s / g \mathrm{BF}$, and computing a leaf from scratch requires leafcost $=$ $(s / g) 2^{g+1} \mathrm{BF}$.

- The intermediate nodes $P[i, j](i \neq j)$ are computed as mentioned before, requiring $2 \mathrm{BF}$.

- The size $N$ of the tree is equal to the number $S$ of signatures we wish to be able to generate with this tree.

1) Cost of signing a message: The signer has to regenerate the private/public key pair, compute the signature, and generate the authentication path for this particular public key. This requires (on average)

precost $+(s / g)\left(2^{g}-1\right)+\frac{\log _{2}(S)}{\log _{2}\left(\log _{2}(S)\right)}(2+$ leafcost $) \mathrm{BF}$.

2) Cost of verifying a signature: The verifier has to check the validity of the signature and of the public key (i.e., verifying the authentication path). This requires (on average)

$$
(s / g)\left(2^{g}-1\right)+2 \log _{2}(S)+(\text { leafcost }- \text { precost }) \mathrm{BF} .
$$

3) Signature size: A signature consists of the public key that was used, the signature itself, and the authentication path to authenticate the public key. The total size of the signature is

$$
2(s / g+1)+\log _{2}(S) \text { blocks. }
$$

\section{Cost Evaluation of HORS Signatures using Merkle Trees}

We propose the following scheme to use Merkle trees for authentication of the HORS public keys:

- In the HORS scheme the private key consists of $t$ random values. These secret values $\sigma_{l}$ are generated using a random seed value $K$ as follows: $\sigma_{l}=A E S_{K}[l]$, with $l=0, \ldots, t-1$ for the first private key, $l=t, \ldots, 2 t-1$ for the second set, etc. The public keys are computed as described in Section IV. Every public key is a leaf pre-images of the Merkle tree. Computing these leaf preimages costs precost $=2 t \mathrm{BF}$.

- The leaves themselves are the result of the OWF $f()$ applied to one of the public keys. This means that

${ }^{3}$ This includes $s / g$ BF for the private key generation and $2(s / g)\left(2^{g}-\right.$ 1) $\mathrm{BF}$ for the public key generation. 
computing a leaf from a leaf pre-image costs $t \mathrm{BF}$, and computing a leaf from scratch requires leafcost $=3 t \mathrm{BF}$.

- The intermediate nodes $P[i, j](i \neq j)$ are computed as mentioned before, requiring $2 \mathrm{BF}$.

- The size $N$ of the tree is equal to $S / r$, with $S$ the number of signatures we wish to be able to generate with this tree, and $r$ the number of signatures that can be placed using the same public key.

1) Cost of signing a message: The signer has to regenerate the private/public key pair, compute the signature, and generate the authentication path for this particular public key. This requires $^{4}$

$$
\text { precost }+\frac{\log (S / r)}{\log _{2}\left(\log _{2}(S / r)\right)}(2+\text { leafcost }) \mathrm{BF} .
$$

2) Cost of verifying a signature: The verifier has to check the validity of the signature and of the public key (i.e., verifying the authentication path). This requires

$$
k+2 \log _{2}(S / r)+(\text { leafcost }- \text { precost }) \text { BF. }
$$

3) Signature size: A signature consists of the public key that was used, the signature itself ( $k$ values), and the authentication path to authenticate the public key. The total size of the signature is

$$
t+k+\log _{2}(S / r) \text { blocks. }
$$

\section{Vi. Authentication with One-way Chains}

In both the basic Lamport-Diffie as het HORS signature scheme the public key is computed by applying a OWF to the private key, which in turn is nothing more than a set of random values. Another way of authenticating these public keys is using one-way chains. Perrig suggests in [18] the use of these one-way chains to authenticate the public keys that are used in the $\mathrm{BiBa}$ (Bins and Balls) one-time signature scheme, but this idea applies equally to other signature schemes. The signer first generates a random root private key $s k_{l}=\left\{x_{(1, l)}, x_{(2, l)}, \ldots, x_{(t, l)}\right\}$. The signer then computes the corresponding public key: $\mathcal{V}_{(i, l)}=f\left(x_{(i, l)}\right)$ for $1 \leq i \leq t$. All other public/private keys are then computed recursively: $\mathcal{V}_{(i, j)}=f\left(\mathcal{V}_{(i, j+1))}\right.$ for $1 \leq i \leq t$ and $j=(l-1) \rightarrow 1$. These one-way chains are depicted in Fig. 4 . The signer divides the time into slots of equal duration. In each time period $i$, the values $\mathcal{V}_{(*, i)}$ make up the active public key. As time advances the public key expires and the next row of values becomes the active public key. Note that the private key in time slot $i$ becomes the public key in the next time slot $i+1$; this is the reason why we introduced the new notation of $\mathcal{V}_{(i, j)}$.

The "top" row of the one-way chains (i.e., the first public key to be used) has to be securely distributed to all possible verifiers. A straightforward solution is to broadcast the first public key $p k_{1}=\left\{\mathcal{V}_{(1,1)}, \mathcal{V}_{(2,1)}, \ldots, \mathcal{V}_{(t, 1)}\right\}$ together with a traditional digital signature such as ECDSA. Once the recipients have obtained this authenticated copy of the first public key, they can trace the one-way chains back until they encounter some value $\mathcal{V}$ in the chain that they know is authentic (in the worst case they have to follow the chain al the way to the first value $\left.\mathcal{V}_{(*, 1)}\right)$. Perrig suggests the following scheme to improve the bootstrapping process (at least for the signer):

\footnotetext{
${ }^{4}$ Signature generation using the HORS scheme is free (see also Section IV).
}

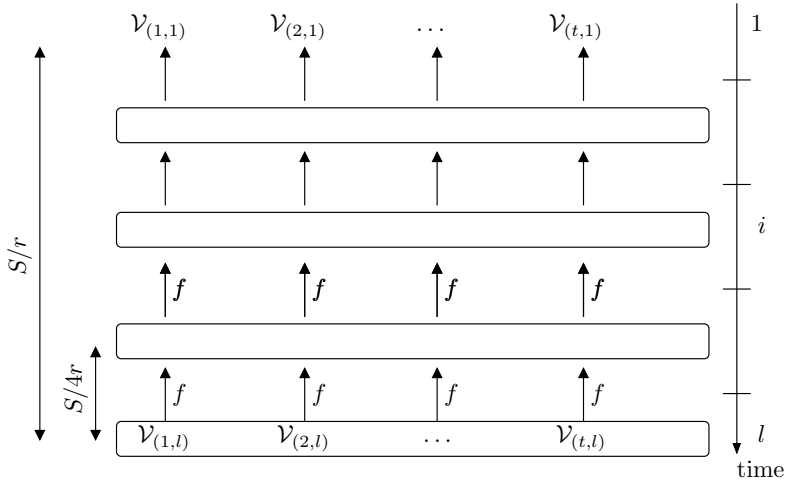

Fig. 4. Authentication of the public keys using one-way chains. The private key in time period $i$ becomes the public key in the next time slot $i+1$.

new recipients start collecting signatures (= different $\mathcal{V}$ 's) as they are broadcasted by the signer. Periodically the signer broadcasts a hash of the public key of one time slot, signed with a traditional digital signature. Once the recipients collect one value of all the one-way chains, they can reconstruct the public key of the specific time slot and validate the signature, and hence validate all subsequent signatures. The "coupon collector" problem predicts that after collection $t \ln (t)$ random $\mathcal{V}$ 's on average the recipients will have one value of every chain [19].

\section{A. Cost evaluation of One-way Chain Authentication}

One-way chains may seem more efficient than Merkle trees as no authentication paths need to be computed, exchanged and verified. On the other hand, the use of one-way chains requires a synchronized clock and puts a limit on the number of messages that can be signed in one time slot (for example, using the Diffie-Lamport scheme it is only possible to sign a single message with one public key).

1) One-way chain generation: Similar to the roots of the Merkle trees, the one-way chains are generated off-line on a powerful device. The result of this process is the top row of the one-way chains (i.e., the first public key $p k_{1}=$ $\left.\left\{\mathcal{V}_{(1,1)}, \mathcal{V}_{(2,1)}, \ldots, \mathcal{V}_{(t, 1)}\right\}\right)$, and at least the bottom row of the chains (i.e., the root private key needed by the signer to regenerate the chains).

If the signer only receives the bottom row, the work load for the first signatures is high because he will have to travel up the chains until he reaches the private key in use at that time period. In order to reduce the work load for the signer, we assume that he also receives 3 more intermediate rows of the chains (see Fig. 4). For the first quarter of the time periods the signer can use the top row to start from to compute the signatures. Once time has advanced beyond this point he can discard this row and start using the next one, etc. Obviously more than four rows can be used for long chains.

2) Signature generation: Unlike when using Merkle trees, there is no need for the signer to regenerate a private key for every signature: signatures are computed using one of the 4 rows of the one-way chains stored in memory by the signer (Fig. 4). Note that in both the basic Lamport-Diffie and HORS signature scheme, the signatures are also elements of the oneway chains. This means that for both schemes, generating a 
signature is nothing more than regenerating a particular row of the one-way chains.

Let $S$ denote the total number of messages that needs to be signed with a chain, $r$ the number of signatures that can be generated with one public key, and $k$ the number of values that make up a signature $(1 \leq k \leq t)$. Independent of the signature scheme used (Lamport-Diffie or HORS) the total signature generation cost is

$4 \times[k r(S / 4 r-1)+k r(S / 4 r-2)+\ldots+k r]=k S^{2} / 8 r$ BF

The average cost per signature is $k S / 8 r \mathrm{BF}^{5}$

3) Signature verification: Assume that the verifiers obtained an authenticated copy of the top row of the chains. Upon reception of a signature ( $=k$ elements of some row), the verifier has to climb up the chains until she reaches a known authenticated value. Once the verifier has checked the authenticity of the received values she only has to memorize the "lowest" value for each chain. This way she will have to compute every value in each chain exactly once. The total signature verification cost is ( $t$ is the number of chains)

$$
t S / r \mathrm{BF} \text { or } t / r \mathrm{BF} \text { per signature. }
$$

\section{B. Cost Evaluation of Basic Lamport-Diffie Signatures using One-way Chains}

We propose the following scheme to use one-way chains to authenticate the Diffie-Lamport public keys:

- Using the basic Diffie-Lamport scheme, a signature on a $s$-bit message requires a public key consisting of $t=$ $s+\left\lceil\log _{2}(s)\right\rceil$ random values $y_{l}=f\left(x_{l}\right)$. Such a set of values make up a row of the chains.

- The chains are computed as described in Section VI.

- On average, a signature will consist of $k=t / 2$ values.

- A public key (a row) can only be used once in this scheme, hence $r=1$, and the length of the chains is equal to the number of signatures $(S)$ we wish to be able to generate.

The cost of signature generation, verification and signature size can be summarized as follows:

- Cost of signing a message:

$$
k S / 8 r=\left(s+\left\lceil\log _{2}(s)\right\rceil\right) S / 16 \mathrm{BF} .
$$

- Cost of verifying a signature: $t / r=s+\left\lceil\log _{2}(s)\right\rceil \mathrm{BF}$.

- Signature size: Public keys do not have to be transmitted when using one-way chains. Hence a signature consists only of the $k$ values, with a size of $k=(s+$ $\left.\left\lceil\log _{2}(s)\right\rceil\right) / 2$ blocks.

\section{Cost Evaluation of HORS Signatures using One-way} Chains

Using one-way chains for authentication of the HORS public keys is straightforward:

- A signature on a $s$-bit message requires a public key consisting of $t$ random values $v_{l}=f\left(s_{l}\right)$, satisfying $s=k \log _{2}(t)$. Such a set of values is one row of the chains.

- The chains are computed as described in Section VI.

${ }^{5}$ Without keeping the four rows in memory, this cost would be $16\left(=4^{2}\right)$ times higher.
- The length of the chains is $S / r$, with $S$ the number of signatures we can generate, and $r$ the number of signatures that can be created using the same public key.

The cost of signature generation, verification and signature size can be summarized as follows:

- Cost of signing a message: $k S / 8 r \mathrm{BF}$.

- Cost of verifying a signature: $t / r \mathrm{BF}$.

- Signature size: Public keys do not have to be transmitted when using one-way chains. Hence a signature consists only of the $k$ values, with a size of $k$ blocks.

\section{COMPARISON}

Table IV summarizes the power consumption of the different signature schemes we evaluated. To facilitate comparison, practical values for the different parameters were chosen. For numerical evaluation, the numbers in Table I are used with the OWF $f()$ based on AES encryption with a 192-bit block size and Elliptic curve calculations in 192-bit fields.

Concerning communications (signature size) we see that ECDSA is most efficient as signatures are only 320 bit, compared to 20-291 blocks for the one-time signature schemes. This makes ECDSA 12-175 times more efficient concerning communications. We also see that using Merkle trees is less efficient than one-way chains as the former requires transmission of the authentication paths.

For verification we see that ECDSA is the worst candidate by a factor 30 compared to the symmetric schemes. The schemes using one-way chains score a little better than the schemes using Merkle trees as the latter require the verification of the authentication path. We also remark that both for communications and verification the differences are almost independent of the number of signatures that are verified or communicated (the cost of Merkle trees only grows with $\left.\log _{2}(S)\right)$.

For signing messages (Fig. 5) the differences grow with the number of signatures. This is because the signature generation cost (per signature) when using one-way chains grows linear with the number of signatures $S$, while when using Merkle trees it only grows with $\log _{2}(S) / \log _{2}\left(\log _{2}(S)\right)$.

Summarizing we see that (1) one-way chains are most efficient for signature verification, (2) ECDSA and Merkle trees are most efficient for signature generation, and (3) ECDSA is the best candidate concerning communications costs. Finally we see that the most demanding task is signature generation, then verification and finally communications.

Finally, Fig. 6 shows a comparison between the different schemes for the case of a single signer and 10 verifiers. We see that up to 15000 signatures the HORS scheme using oneway chains is the cheapest option, when more than 15000 signatures are required the best option becomes Lamport-Diffie signatures using Merkle trees. The ECDSA is 2.5-7 times more expensive than Lamport-Diffie signatures using Merkle trees, but obviously much easier to menage.

\section{CONCLUSIONS}

In this paper we have investigated the power consumption of several digital signature schemes. Our evaluation includes all aspects of using these schemes, i.e., key generation, signature generation and verification, and communications. These evaluations result in algebraic expressions for these different 
aspects. Including all these cost elements, we conclude that, on the hardware/software implementation we used as an example, the ECDSA only requires 2.5-7 times more power than the cheapest scheme based on symmetric techniques. Considering the fact that using ECDSA is much easier to manage (short public key, private key can be used multiple times, etc.) ECDSA might prove be the best solution overall in many scenarios.

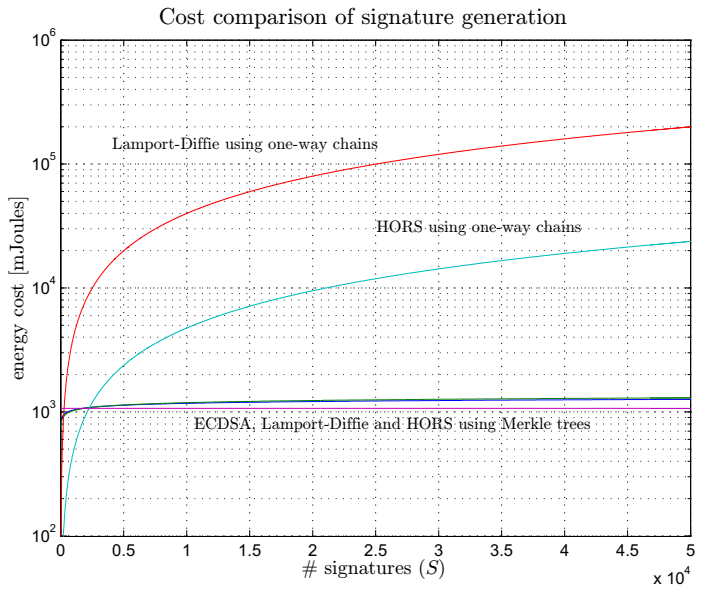

Fig. 5. Energy consumption of signature generation in the different schemes.

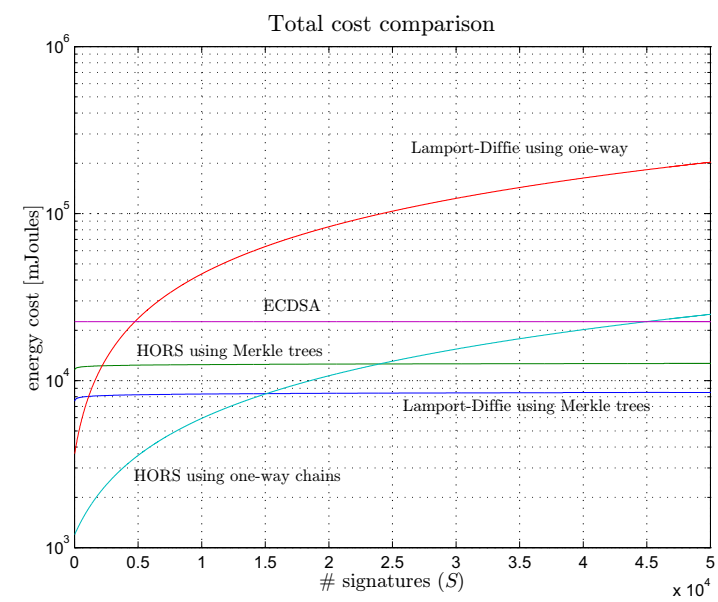

Fig. 6. Comparison of energy consumption of the different schemes. This setting assumes one signer and ten verifiers.

\section{REFERENCES}

[1] "Center for embedded networked sensing (CENS)." [Online]. Available: http://cens.ucla.edu/

[2] "PicoRadio, the Berkeley wireless research center PicoRadio project." [Online]. Available: http://bwrc.eecs.berkeley.edu/Research/Pico_Radio/

[3] "MIT $\mu$ AMP ( $\mu$-adaptive multi-domain power aware sensors) project." [Online]. Available: http://www-mtl.mit.edu/research/icsystems/uamps/

[4] University of California, "Wireless integrated network sensors (WINS)." [Online]. Available: http://www.janet.ucla.edu/WINS/

[5] W. Ye, J. Heidemann, and D. Estrin, "An energy-efficient MAC protocol for wireless sensor networks," in Proceedings of the 21st International Annual Joint Conference of the IEEE Computer and Communications Societies (INFOCOM '02). IEEE, 2002.
TABLE IV

SumMARY OF Digital SignATURE COSTS. Message HASH SizE $s=160$.

\begin{tabular}{|l|l|}
\hline \multicolumn{2}{|l|}{ Lamport-Diffie using Merkle trees $\left(g=2, S=2^{16}\right)$} \\
\hline$p k$ generation & off-line \\
Signing & $800+652 \log _{2}(S) / \log _{2}\left(\log _{2}(S)\right) \mathrm{BF}=3368 \mathrm{BF}$ \\
Verification & $320+2 \log _{2}(S) \mathrm{BF}=332 \mathrm{BF}$ \\
$p k$ size & root node $=1$ block \\
Signature size & $162+\log _{2}(S)=178$ blocks \\
\hline \hline Lamport-Diffie using one-way chains $\left(g=2, S=2^{16}\right)$ \\
\hline$p k$ generation & off-line \\
Signing & $(168 / 16) S \mathrm{BF}=688128 \mathrm{BF}$ \\
Verification & $168 \mathrm{BF}$ \\
$p k$ size & top row $=168$ block \\
Signature size & 84 blocks \\
\hline \hline HORS using Merkle trees $\left(k=20, t=256, r=2, S=2^{16}\right)$ \\
\hline$p k$ generation & off-line \\
Signing & $512+770 \log _{2}(S / 2) / \log _{2}\left(\log _{2}(S / 2)\right) \mathrm{BF}=3520 \mathrm{BF}$ \\
Verification & $276+2 \log _{2}(S / 2) \mathrm{BF}=306 \mathrm{BF}$ \\
$p k$ size & root node $=1$ block \\
Signature size & $276+\log _{2}(S)=291$ blocks \\
\hline \hline HORS using one-way chains $\left(k=20, t=256, r=2, S=2^{16}\right)$ \\
\hline$p k$ generation & off-line \\
Signing & $(5 / 4) S$ BF $=81920 \mathrm{BF}$ \\
Verification & $128 \mathrm{BF}$ \\
$p k$ size & top row $=256$ block \\
Signature size & 20 blocks \\
\hline
\end{tabular}

[6] I. F. Akyildiz, W. Su, Y. Sankarasubramaniam, and E. Cayirci, "A survey on sensor networks," IEEE Communications Magazine, vol. 40, no. 8, pp. 102-114, Aug. 2002.

[7] M. Jakobsson and D. Pointcheval, "Mutual authentication for low-power mobile devices," in Proceedings of Financial Cryptography (FC '01), ser. Lecture Notes in Computer Science, P. Syverson, Ed., vol. 2339. Springer, 2001, pp. 178-195.

[8] E. Bresson, O. Chevassut, A. Essiari, and D. Pointcheval, "Mutual authentication and group key agreement for low-power mobile devices," in Proceedings of The Fifth IEEE International Conference on Mobile and Wireless Communications Networks (MWCN '03). IEEE, 2003.

[9] L. Lamport, "Constructing digital signatures from a one way function," SRI International, Tech. Rep. CSL-98, Oct. 1979.

[10] L. Reyzin and N. Reyzin, "Better than BiBa: Short one-time signatures with fast signing and verifying," in Proceedings of the 7th Australian Conference on Information Security and Privacy, ser. Lecture Notes in Computer Science, J. Seberry, Ed., vol. 2384. Springer, 2002, pp. 144 153.

[11] D. Johnson and A. Menezes, "The Elliptic Curve Digital Signature Algorithm (ECDSA)," Dept. of C\&O, University of Waterloo, Canada," Technical Report CORR 99-34, Aug. 1999, updated: 2000/02/24.

[12] A. Hodjat and I. Verbauwhede, "The energy cost of secrets in adhoc networks," in Proceedings of the IEEE CAS Workshop on Wireless Communications and Networking. IEEE, 2002.

[13] "Wireless Sensing Networks at Rockwell Scientific." [Online]. Available: http://wins.rockwellscientific.com/

[14] A. Menezes, P. van Oorschot, and S. Vanstone, Handbook of Applied Cryptography. CRC Press, 1997.

[15] I. F. Blake, G. Seroussi, and N. P. Smart, Elliptic Curves in Cryptography. Cambridge University Press, July 1999.

[16] R. C. Merkle, "A certified digital signature," in Advances in Cryptology - CRYPTO '89, ser. Lecture Notes in Computer Science, G. Brassard Ed., vol. 435. Springer, 1990, pp. 218-238.

[17] M. Jakobsson, T. Leighton, S. Micali, and M. Szydlo, "Fractal Merkle tree representation and traversal," in Topics in Cryptology - RSA Conference Cryptographers' Track (RSA-CT 'O3), ser. Lecture Notes in Computer Science, vol. 2612. Springer, 2003.

[18] A. Perrig, "The BiBa one-time signature and broadcast authentication protocol," in Proceedings of the 8th ACM Conference on Computer and Communications Security (CCS '01). ACM Press, New York, NY, USA, 2001.

[19] M. Finkelstein, H. G. Tucker, and J. A. Veeh, "Confidence intervals for the number of unseen types," Statistics and Probability Letters, vol. 37, pp. 423-430, 1998.

Published in Proceedings of the 2005 IEEE International Conference on Wireless and Mobile Computing, Networking and Communications (IEEE WiMob 2005), Volume 1, pp. 79-86, IEEE, 2005. 